\title{
Precoder-Aided Iterative Detection Assisted Multilevel Coding and Three-Dimensional EXIT-Chart Analysis
}

\author{
R. Y. S. Tee, S. X. Ng and ${ }^{1}$ L. Hanzo \\ School of ECS, University of Southampton, SO17 1BJ, UK. \\ Tel: +44-23-8059 3125, Fax: +44-23-8059 4508 \\ Email: ${ }^{1}$ lh@ecs.soton.ac.uk, http://www-mobile.ecs.soton.ac.uk
}

\begin{abstract}
A novel three-dimensional (3D) EXIT chart is developed for investigating the iterative behaviour of Multilevel Coding (MLC) invoking Multistage Decoding (MSD). The extrinsic information transfer characteristics of both the symbol-to-bit demapper used and those of the differentprotection constituent decoders suggest that potential improvements can be achieved by appropriately designing the demapper. The proposed 3D EXIT chart is then invoked for studying the precoder-aided multilevel coding scheme employing both MSD and Parallel Independent Decoding (PID) for communicating over AWGN and uncorrelated Rayleigh fading channels with the aid of 8PSK modulation. At BER $=10^{-5}$, the precoder was capable of enhancing the achievable $E_{b} / N_{0}$ performance by $0.5 \mathrm{~dB}$ to $2.5 \mathrm{~dB}$ over AWGN and Rayleigh channels, respectively.
\end{abstract}

\section{INTRODUCTION}

Multilevel Coding (MLC) was introduced by Imai and Hirawaki [1] as a bandwidth efficient coded modulation scheme, which was designed for protecting each bit of non-binary modulation schemes with the aid of potentially different-rate binary codes. The so-called capacity rules have been proposed [2] for choosing appropriate coding rates that are capable of approaching the channel capacity with the aid of Multistage Decoding (MSD), while Parallel Independent Decoding (PID) has been employed as a design alternative for the sake of reducing the associated decoding delay. However, the iterative decoding behaviour of MLC schemes depends on the mutual information transfer characteristics of both the decoders as well as on those of the demapper used for conveying the demodulated bits to the constituent decoders.

With the objective of studying the iterative detection aided performance of MSD assisted MLCs, we propose a novel threedimensional (3D) extrinsic information transfer (EXIT) [3] chart for investigating the effects of different symbol-to-bit demapper characteristics. In the recent past, different constellation labeling strategies have been employed in the context of MLC for the sake of increasing either the Euclidean distance or the Hamming distance associated with the different modulation phasor points in order to achieve a better iterative detection

The financial support of the European Union under the auspices of the Phoenix and Newcom projects and that of the EPSRC, UK is gratefully acknowledged. performance with the aid of optimized bit-to-symbol mappers / demappers [2] [4] [5]. In this paper, instead of optimizing the modem constellation labelling, we introduce a serially concatenated unity-rate code [6] having a recursive structure as a precoder in the context of MLC schemes for the sake of enhancing the demapper's convergence characteristics. Furthermore, we will benchmark our MLC MSD scheme against the Parallel Independent Decoding (PID) [2] scheme, which exhibits a significantly reduced decoding delay.

The rest of this contribution is organized as follows. Section 2 provides an overview of the system considered, while our novel 3D EXIT chart is invoked in Section 3 for the sake of characterizing the system's iterative convergence behaviour. Section 4 quantifies the performance of our pre-coded MLC scheme, while our conclusions are presented in Section 5.

\section{SYSTEM OVERVIEW}

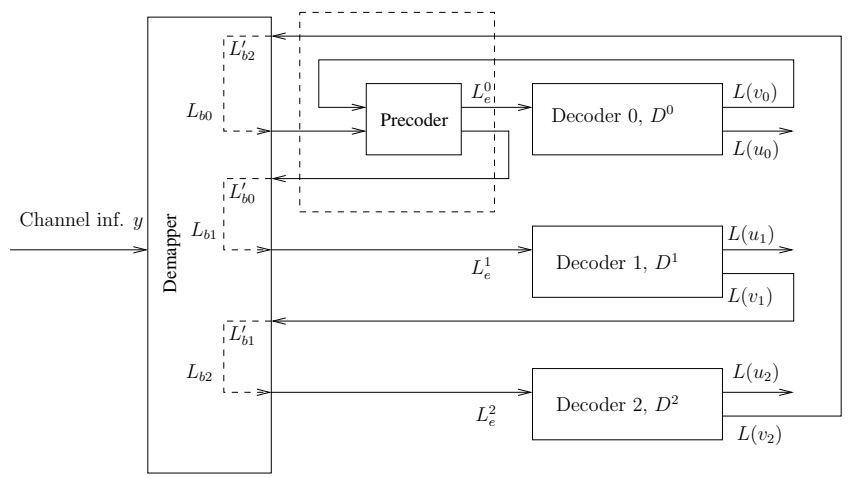

Figure 1: MSD Decoder of the 8PSK modulation based precoderaided MLC scheme.

Figure 1 and 2 outline our MSD and PID schemes designed for operating in conjunction with 8PSK modulation, respectively. The notations $L\left(u_{i}\right)$ and $L\left(v_{i}\right)$ represent the output LLRs of the decoders for the original information bits and for the MLC-encoded bits, respectively. The subscript $i$ represents the index of the different-protection bits $b 0, b 1$ and $b 2$, while $L_{e}^{i}$ denotes the extrinsic LLR generated at the output of the inner demapper. The rectangle drawn in dashed lines in both Figures 1 and 2 represents the precoder schemes constituted by unity-rate recursive encoders inserted after the demapper. In Figure $1, L_{b i}$ denotes the associated information bits' LLRs for 


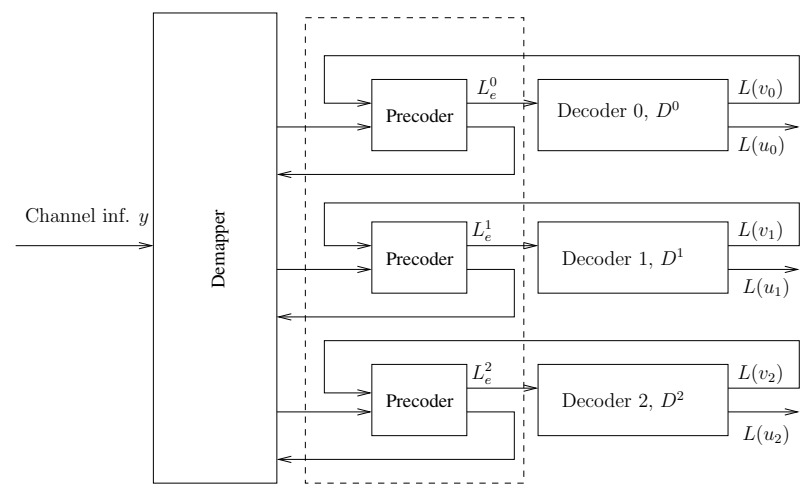

Figure 2: PID Decoder of the 8PSK modulation based precoderaided MLC scheme.

the corresponding decoder $D^{i}$, while $L_{b i}^{\prime}$ denotes the a priori LLRs forwarded by the other decoder $D^{i}$ to the input of the inner demapper.

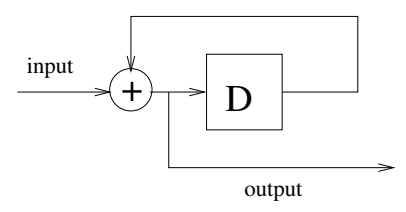

(a)

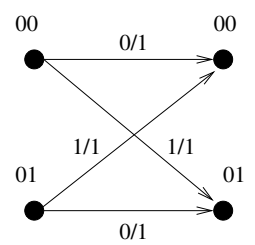

(b)
Figure 3: Unity rate memory-1 precoder

To elaborate a little further, in the MSD decoder of Figure 1 the a priori information is fed by the lower-protection decoder to the higher-protection scheme. Each of the bits $b 0, b 1$ and $b 2$ is decoded by the corresponding decoder, namely by $D^{0}, D^{1}$ and $D^{2}$. The decoder $D^{i}$ processes both the received information bits of $L_{b i}$ as well as the a priori information provided by the other decoders and conveyed by the inner demapper seen in Figure 1. By contrast, the PID structure shown in Figure 2 does not make use of the decisions carried out at other protection levels. Instead, each decoder $D^{i}$ processes the a priori knowledge in a parallel and independent manner. Hence, this potentially results in a reduction of the associated processing delay.

Figure 3 portrays the unity-rate code employed in our precoder-aided MLC scheme, where $D$ is a shift register stage and $\oplus$ represents the modulo- 2 operation. Figure 3(b) shows the trellis diagram of the precoder. The trellis transitions are denoted by $c_{i} / x_{i}$, where $c_{i}$ denotes the input of the precoder at time $i$, while $x_{i}$ indicates the corresponding precoder output.

In the system advocated, we employ convolutional codes as our component, where the individual coding rates of the MLC MSD and MLC PID schemes are 1/3, 3/4, 11/12 [7] and $1 / 2,3 / 4$ and $3 / 4$ [2], respectively. Since the specific coding rates that are readily available for convolutional codes are constrained, we do not follow the exact capacity rules proposed in [2] for adjusting the MLC scheme's coding rate. The resultant effective throughput of the 8PSK system considered becomes
2 bits per symbol. All system parameters are summarized in Table 1.

\begin{tabular}{|l||c|c|c|}
\hline Coding rate & $R_{0}$ & $R_{1}$ & \multicolumn{1}{c|}{$R_{2}$} \\
\hline \hline MSD & $1 / 3$ & $3 / 4$ & $11 / 12$ \\
PID & $1 / 2$ & $3 / 4$ & $3 / 4$ \\
\hline Precoders' trellis states & \multicolumn{3}{|c|}{00 and 01 } \\
Modulation & 8PSK \\
Mapping type & Set Partitioning (SP) \\
Interleaver length & \multicolumn{1}{|c|}{1800 symbols } \\
\hline
\end{tabular}

Table 1: System parameters.

\section{EXIT CHART BASED CONVERGENCE ANALYSIS}

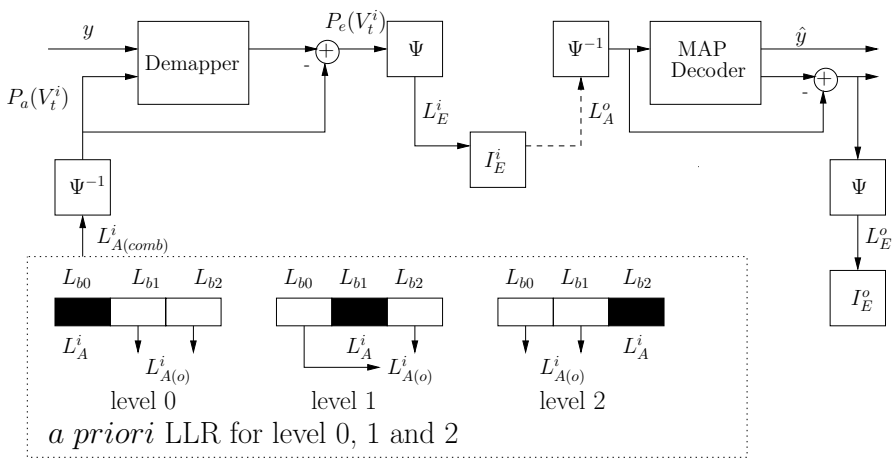

Figure 4: EXIT chart generation for the MSD of 3-level MLC when using 8PSK and three en(de)coders.

In this section, we introduce a 3D EXIT chart for the sake of analyzing the iterative convergence behaviour of the MLC MSD scheme considered, where the demapper and decoder are referred to as the inner and outer codes. Figure 4 shows the schematic of generating the 3D EXIT chart. Specifically, $L_{b}^{a}$ represents the LLR values, where the superscript $a$ denotes the inner $(i)$ or outer $(o)$ codes, while the subscript $b$ denotes the input a priori $(A)$ or output extrinsic $(E)$ information. The variables $L_{b 0}, L_{b 1}$ and $L_{b 2}$ are independent Gaussian distributed LLRs generated for bits 0,1 and 2, respectively. Furthermore, $\Psi$ and $\Psi^{-1}$ denote the LLR-to-symbol probability and symbol probability-to-LLR conversion. The arrow drawn in dashed line represents the extrinsic LLR demapper output, which becomes the LLR input of the decoder after demapping. The filled black box represents the a priori LLR of the associated information bit, while the hollow box denotes the a priori LLR of the other decoders' bits. Both $I_{E}^{i}$ and $I_{E}^{o}$, which denote the mutual information accruing from the inner and outer codes are used for plotting the EXIT chart.

In order to generate the 3D EXIT chart for the MLC MSD scheme seen in Figure 1 for an 8PSK modulated system, we model the LLRs $L_{A}^{i}$ and $L_{A(o)}^{i}$ by independent Gaussian distributed random variables [3] at MLC protection level 0, 1 and 2, as shown in Figure 4. Considering the example of protection level 0, the LLR $L_{A}^{i}$ associated with the black box in Figure 4 is generated from $L_{b 0}$. The a priori LLRs $L_{b 1}$ and $L_{b 2}$ gener- 
ated by the other decoders and indicated by the hollow boxes in Figure 4 are then computed along with their individual average values for the sake of obtaining the combined a priori LLR of $L_{A(0)}^{i}$. Similar operations are carried out at the decoder of protection level 1 and level 2, each having the corresponding information bit represented by the black box at the appropriate position. The mutual information corresponding to $L_{A}^{i}$, $L_{A(o)}^{i}$ and $L_{A}^{o}$ is represented by $I_{A}^{i}, I_{A(o)}^{i}$ and $I_{A}^{o}$, respectively.

In Figure 5, the EXIT plane marked with the mesh of triangles denotes the extrinsic information transfer characteristics of the inner demapper, while the plane represented by the mesh of rectangles characterizes the extrinsic information of the outer MAP decoder. The thick zig-zag shaped lines seen in Figure 5 represent the decoding trajectory evolving within the 3D tunnel, constituted by the inner demapper's and the outer decoder's EXIT planes. If the trajectory succeeds in converging to the point $Q(1,1,1)$, the highest possible iteration gain is reached. We observe that the decoding trajectory of protection level 0 fails to reach this point owing to its deficient demapper characteristics. Hence, in order to improve the iterative decoding convergence of the overall MLC system, we have to improve the demapper characteristic at protection level 0 . This can be achieved by introducing the precoders [6] shown in the dash-line boxes of Figure 1 and 2 .

The beneficial effect of precoding on the attainable decoding convergence has been demonstrated by Narayanan in [8], where the recursive precoder of $g(D)=1 \oplus D^{R}$ was shown to attain a substantial gain. In the precoder's generator polynomial, $R$ represents an integer which corresponds to the number of shift register stages employed in the precoder structure. Here, we employ a low-complexity rate-1 precoder having the generator polynomial $g(D)=1+D$ without adding further redundancy to the inner code's demapper, which allows us to maintain the inner code's original coding rate. Figure 3 demonstrates the implementational simplicity of the precoder, which has a 2-state trellis.

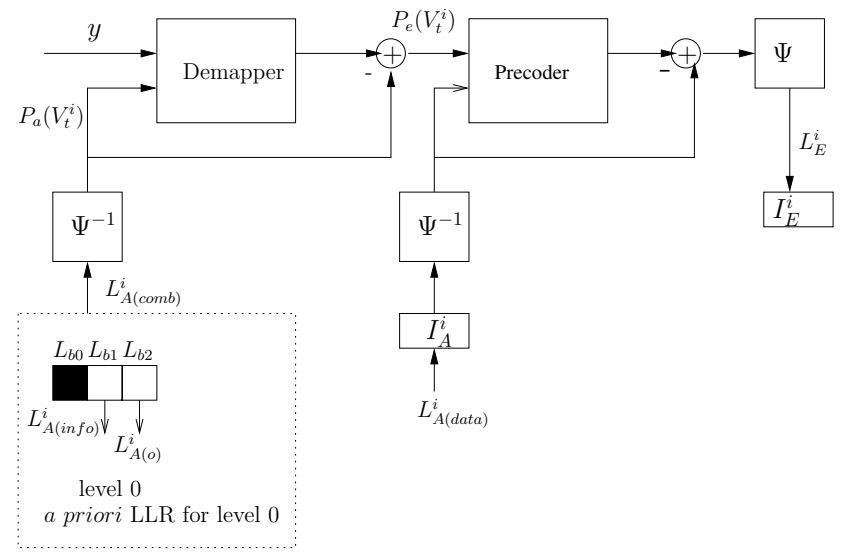

Figure 6: EXIT chart generation for the MSD MLC scheme of Figure 1 , when using 8PSK and three en(de)coders.

The schematic of generating the 3D EXIT plane for protection level 0 of the precoder-aided MSD is shown in Figure 6. The 3D EXIT plane of Figure 5 shows that the inner demapper characteristics are only affected by the information bits provided by the LLRs of the other $L_{A(o)}^{i}$ decoders, regardless of its own intrinsic LLR $L_{A}^{i}$. Hence in Figure 6, $L_{A(i n f o)}^{i}$ would only be regarded as dummy information and would not be exploited in the 3D EXIT chart for the sake of reducing its dimensions. In other words, the inner code's EXIT plane will not be affected by varying the values of $L_{A(i n f o)}^{i}$. The LLR $L_{A(\text { data })}^{i}$ is generated by the outer MAP decoder and input to the precoder. Therefore, the LLRs $L_{A(o)}^{i}, L_{A(\text { data })}^{i}$ and $L_{E}^{i}$ corresponding to the mutual information $I_{A(o)}^{i}, I_{A}^{i}$ and $I_{E}^{i}$ are used for plotting the inner demapper's EXIT characteristic. The inner EXIT characteristic plane is a function of $I_{E}^{i}=$ $f\left(I_{A}^{i}, I_{A(o)}^{i}\right)$.

Figure 7 portrays the 3D EXIT chart employing the precoder. The EXIT planes marked with the mesh of triangles and dashed lines characterize the precoded demapper at $\mathrm{SNR}=6 \mathrm{~dB}$ and $\mathrm{SNR}=4 \mathrm{~dB}$, respectively, while the mesh of rectangles represents the outer MAP decoder's EXIT characteristics. Observe in Figure 7 that there is no iteration gain at $\mathrm{SNR}=4 \mathrm{~dB}$, since the EXIT plane is trapped below the MAP decoder's plane. By contrast, at $\mathrm{SNR}=6 \mathrm{~dB}$ we are able to obtain an open tunnel between the two EXIT planes, which eventually reaches the point of decoding convergence at $Q(1,1,1)$. Note that the trajectory evolves between the two planes and the a priori knowledge extracted from the associated information bits affects the extrinsic information as opposed to the MLC scheme characterized in Figure 5. More explicitly, with the aid of the precoder's a priori information, the trajectory no longer evolves in parallel to the $y$ axis, as seen in Figures 5(a), 5(b) and 5(c), but instead it evolves in an angle as a function of both $I_{A}^{i}\left(x\right.$ axis) and $I_{A(o)}^{i}(y$ axis), as shown in the dotted section of the trajectory seen in Figure 7. We now continue our discourse by introducing the precoder in the PID aided MLC scheme shown in Figure 2.

\section{SIMULATION RESULTS}

In this section we characterize the BER performance of the precoder-aided iterative MLC scheme of Figure 1 and 2 using 8PSK as well as Set Partition (SP) [2] based mapping. Figure 8 shows the attainable BER versus $E_{b} / N_{0}$ performance, when communicating over an AWGN channel. The precoder-aided MLC scheme of Figure 1 is outperformed by the MLC scheme at an SNR below $5 \mathrm{~dB}$. This is, because the precoded scheme does not exhibit any iteration gain for SNRs below $4 \mathrm{~dB}$, as shown in the 3D EXIT plane of Figure 7 with the aid of the plane indicated by the dotted dashed lines. Once the EXIT plane has an open tunnel at the higher SNR of $6 \mathrm{~dB}$, the associated BER performance is substantially improved, with the advent of introducing precoder as observed in Figure 8. Note that the precoder-aided scheme exhibits a 'turbo-like' behaviour in Figure 8 and exhibits no error floor, as opposed to the conventional MLC scheme. A more significant BER performance improvement can be observed in Figure 9, when communicating over an uncorrelated Rayleigh fading channel. 


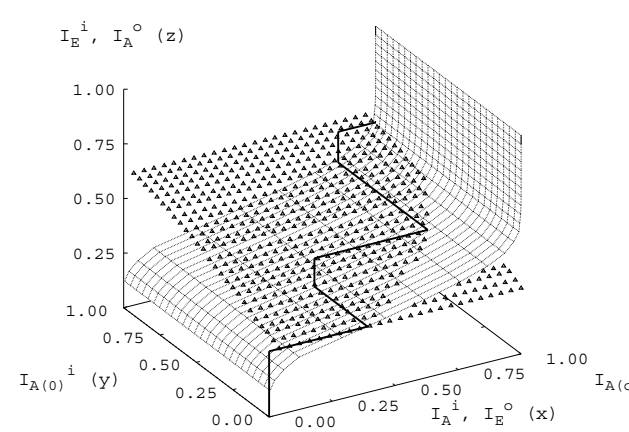

(a) Level 0

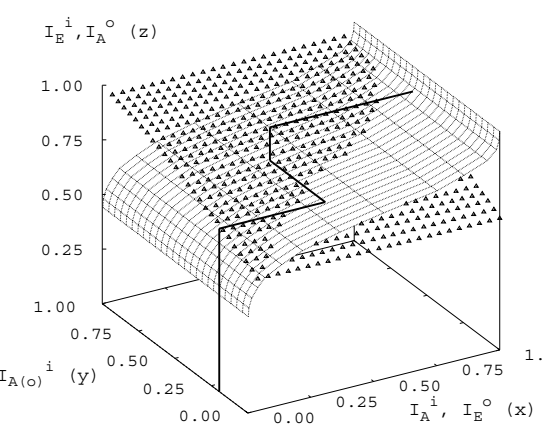

(b) Level 1

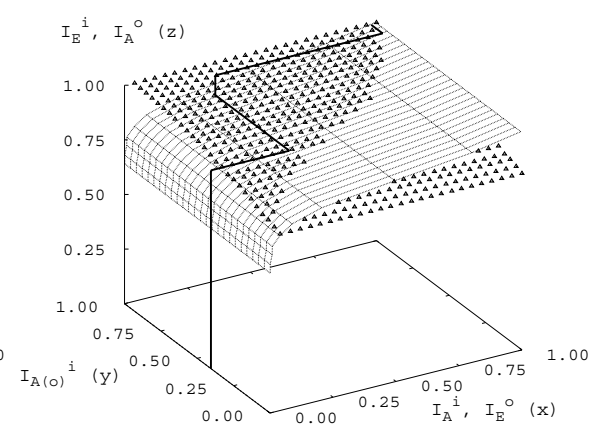

(c) Level 2

Figure 5: 3D EXIT Chart for Level 0, Level 1 and Level 2 of the MLC scheme at SNR $=4 \mathrm{~dB}$.

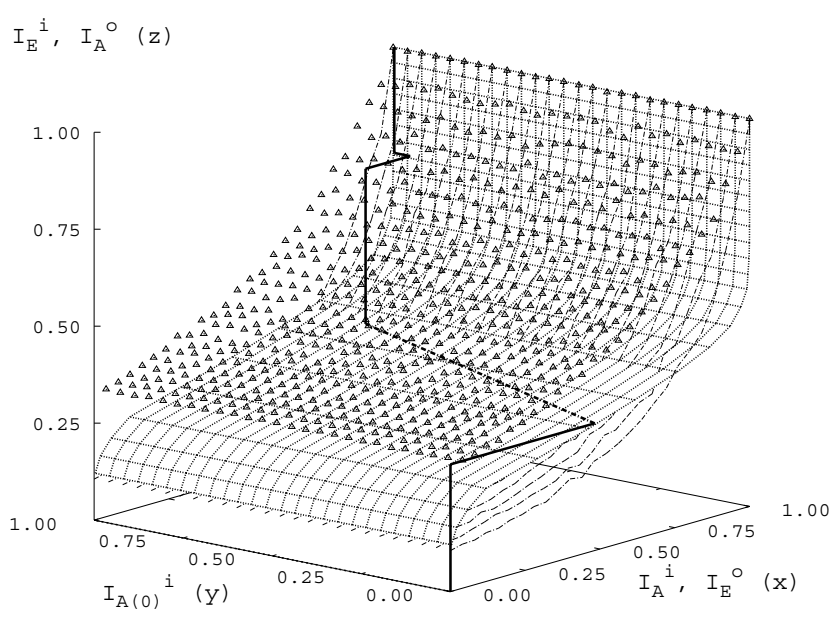

Figure 7: 3D EXIT chart for protection level 0 of the precoder-aided MLC scheme of Figure 1 at SNR $=4 \mathrm{~dB}$ (dotted dashed lines) and $\mathrm{SNR}=6 \mathrm{~dB}$ (mesh of triangles).

Figures 10 and 11 illustrate the attainable BER performance of the precoder-aided MLC PID scheme communicating over both AWGN and uncorrelated Rayleigh fading channels. At $\mathrm{BER}=10^{-5}$, the precoder-aided MLC scheme exhibits a significant coding advantage of about $2 \mathrm{~dB}$ in AWGN channels and about $5 \mathrm{~dB}$ in uncorrelated Rayleigh channels. Note that PID becomes capable of outperforming MSD in the precoderaided MLC scheme, as the benefit of its higher iteration gain. This is due to the fact that the decision errors of the lower protection levels may spread to the higher levels in MSD. Furthermore, SP-based mapping, which maximizes the Euclidean distance of phasor constellation points for the sake of obtaining an iteration gain, performs better in AWGN channels. In Figure 12, we further compare the precoder-aided MLC PID scheme to other coded modulation schemes having the same expressed complexity in terms of the number of trellis states

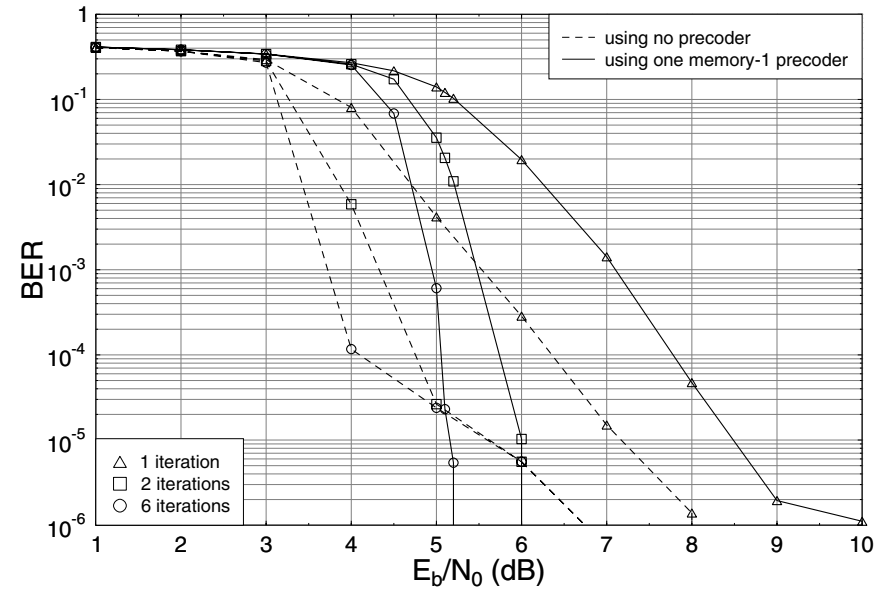

Figure 8: BER versus $E_{b} / N_{o}$ performance of the conventional and precoder-aided 8PSK modulated MLC MSD scheme of Figure 1, communicating over an AWGN channel.

and communicating over uncorrelated Rayleigh fading channels. Our precoder-aided MLC scheme exhibits a better BER performance associated with a coding advantage of $2.5 \mathrm{~dB}$ at $\mathrm{BER}=10^{-5}$ compared to the best-performing BICM-ID coded modulation scheme, although it is outperformed by TTCM, both of which were detailed in [9].

\section{CONCLUSIONS}

In conclusion, this paper provides an insight into the iterative decoding convergence behaviour of precoder-aided MLC MSD and MLC PID schemes. We proposed the novel tool of 3D EXIT charts for the sake of investigating the iterative convergence of precoder-aided MLC schemes. Our simulation results outlined in Figures 8, 9, 10 and 11 illustrate that the precoder-aided iterative MLC scheme achieves a significant BER performance improvement both in AWGN and uncorrelated Rayleigh channels, which is achieved without reducing the overall coding rate and without significantly increasing the complexity of the conventional MLC scheme. Alter- 


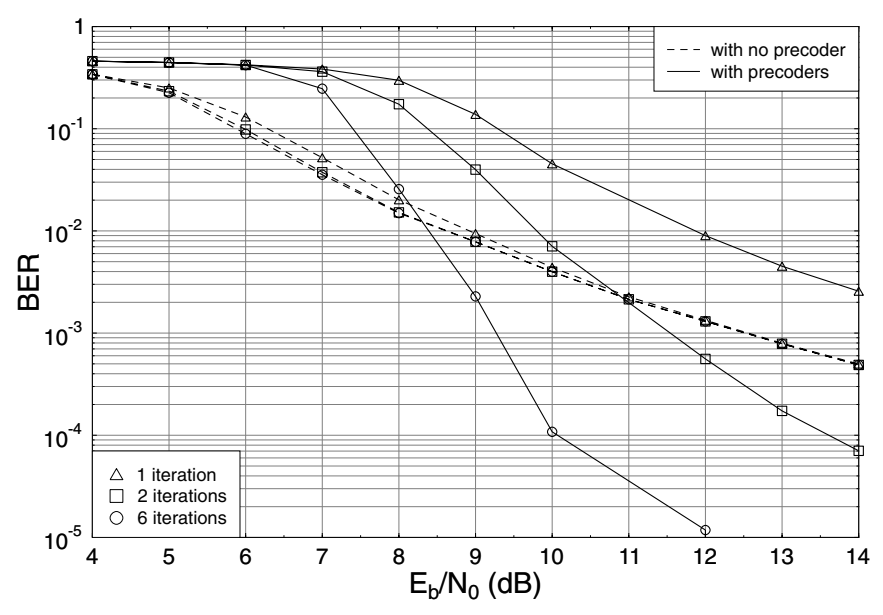

Figure 9: BER versus $E_{b} / N_{o}$ performance of both conventional and precoder-aided 8PSK modulated MLC MSD scheme, communicating over uncorrelated Rayleigh channel.

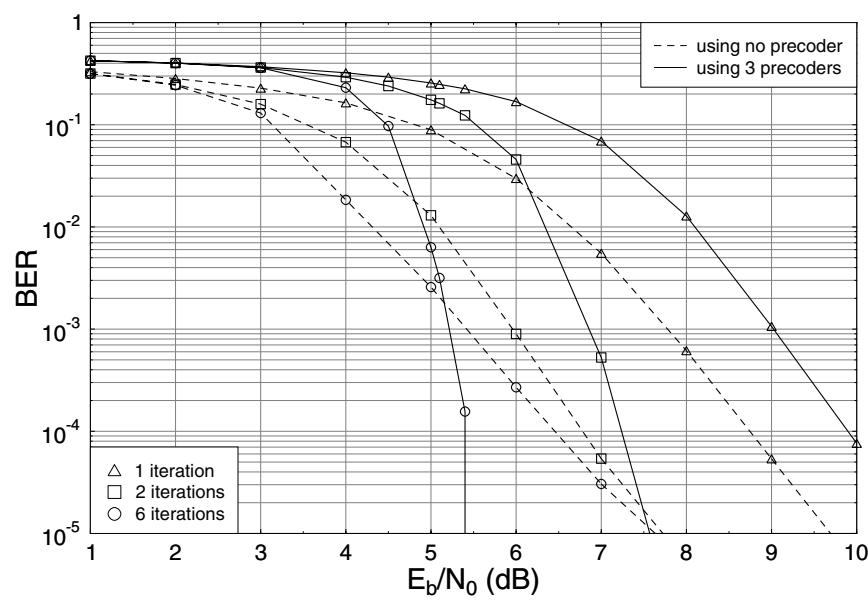

Figure 10: BER versus $E_{b} / N_{o}$ performance of both conventional and precoder-aided 8PSK modulated MLC PID scheme, communicating over AWGN channel.

natively, at $\mathrm{BER}=10^{-5}$ the precoder was capable of enhancing the achievable $E_{b} / N_{0}$ performance by $0.5 \mathrm{~dB}$ and $5 \mathrm{~dB}$, when communicating over AWGN and Rayleigh channels, respectively. Our future research investigates the iterative decoding performance of combined MLCs and Generalized LowDensity Parity-Check (GLDPC) codes.

\section{REFERENCES}

[1] H. Imai and S. Hirawaki, "A New Multilevel Coding Method Using Error Crrecting Codes," IEEE Transactions on Information Theory, pp. 371377, May 1977.

[2] U. Wachsmann, R. F. H. Fischer and J. B. Huber, "Multilevel Codes: Theoretical Concepts and Practical Design Rules," IEEE Transaction on Information Theory, vol. 45, pp. 1361-1391, July 1999.

[3] S. ten Brink, "Convergence Behavior of Iteratively Decoded Parallel Concatenated Codes," IEEE Transactions On Communications, pp. 17271737, October 2001.

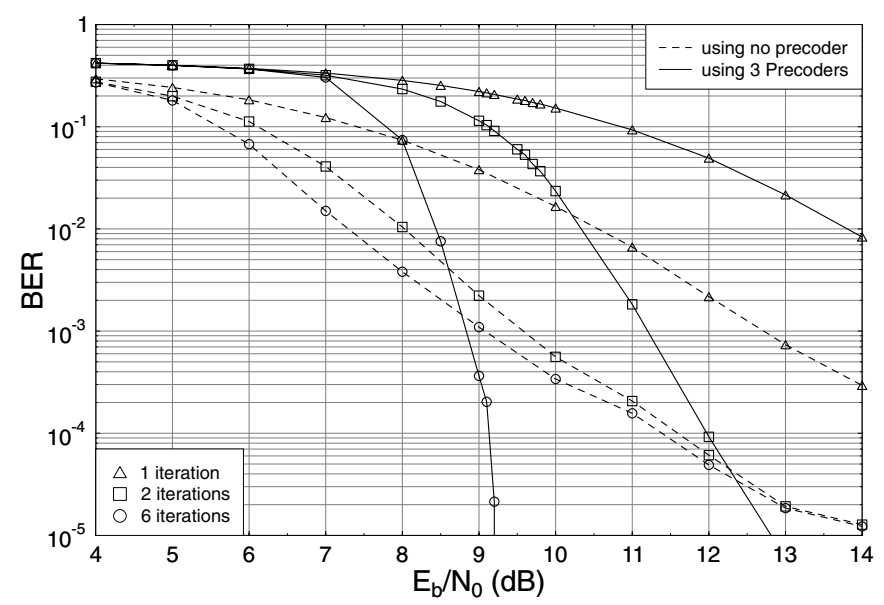

Figure 11: BER versus $E_{b} / N_{o}$ performance of both conventional and precoder-aided 8PSK modulated MLC PID schemes, communicating over uncorrelated Rayleigh channels.

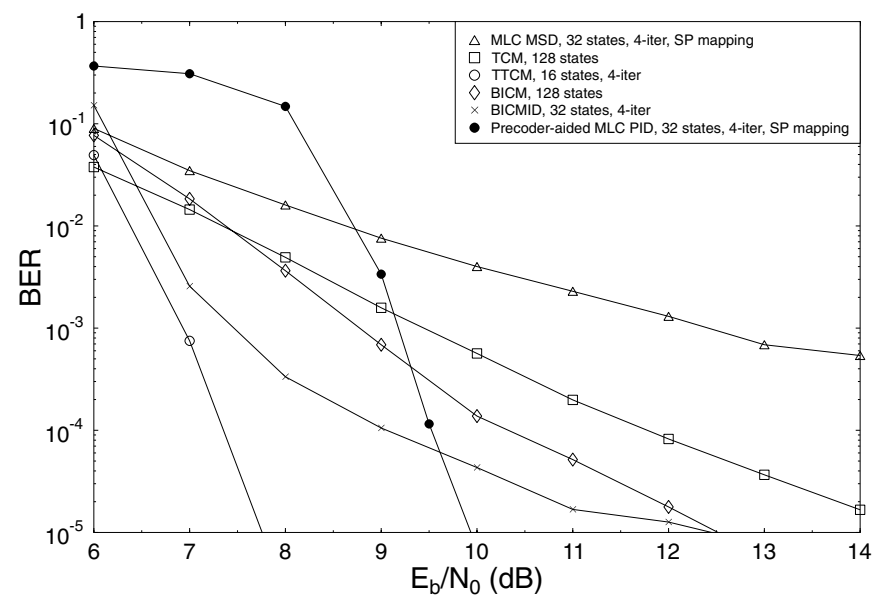

Figure 12: BER versus $E_{b} / N_{o}$ performance of various coded modulation schemes having MAP decoding complexity associated with 128-trellis states, when communicating over uncorrelated Rayleigh channels using 8PSK modulation.

[4] D. F. Yuan, P. Zhang, Q. Wang and W. E. Stark, "A Novel Multilevel Codes With 16QAM," IEEE Wireless Communications and Networking Conference, pp. 260-263, 2002.

[5] F. Schreckenbach and G. Bauch, "EXIT charts for iteratively decoded multilevel modulation," 12th European Signal Processing Conference (EUSIPCO), 2004.

[6] D. Divsalar, S. Dolinar and F. Pollara, "Serial Concatenated Trellis Coded Modulation with Rate-1 Inner Code," IEEE Global Telecommunications Conference, pp. 777-782, Nov. 2000.

[7] M. Isaka and H. Imai, "On the Iterative Decoding of Multilevel Codes," IEEE Journal on Selected Areas in Comms, vol. 19, pp. 935-943, May 2001.

[8] K. R. Narayanan, "Effect of Precoding on the Convergence of Turbo Equalization for Partial Response Channels," IEEE Journal on Selected Areas in Communications, pp. 686-698, Apr. 2001.

[9] L. Hanzo, T. H. Liew and B. L. Yeap, Turbo Coding, Turbo Equalisation and Space Time Coding for Transmission over Wireless channels. New York, USA: John Wiley IEEE Press, 2002. 